\title{
Correction to: Incorporating Colorectal Cancer Genetic Risk Assessment into Gastroenterology Practice
}

Benjamin Stern, $D 0^{1, *}$

Thomas McGarity, MD

Maria Baker, PhD, $M S^{2}$

\author{
Address \\ ${ }^{*}, 1$ Division of Gastroenterology and Hepatology, Penn State Health Milton S. \\ Hershey Medical Center, 200 Campus Dr., Suite 2400|Entrance 4, Hershey, PA, \\ 17033, USA \\ Email: bstern@pennstatehealth.psu.edu \\ ${ }^{2}$ Division of Hematology Oncology, Penn State Health Milton S. Hershey Medical \\ Center, Penn State Cancer Institute, 400 University Dr., Hershey, PA, 17033, USA
}

Published online: 23 December 2019

(C) Springer Science+Business Media, LLC, part of Springer Nature 2019

The online version of the original article can be found at https://doi.org/10.1007/s11938-019-00267-w

\section{Correction to: Curr Treat Options Gastro https://doi.org/10.1007/s11938-019-00267-w}

The original article unfortunately contained a mistake. On page 8, Table 2, the row with "POLE POLD1" has been shifted right so all the columns are not correct.

The original version has been corrected. 